\title{
Evaluation Method of Decision-making Efficiency on Control Information System Oriented Multi- Cooperated*
}

\author{
Zhengxian Wei ${ }^{1,2}$, Min Song ${ }^{3}$, Bo Li ${ }^{1}$ \\ ${ }^{1}$ System Engineering Research Institute, Beijing, China \\ ${ }^{3}$ Beijing Foreign Studies University, Beijing, China \\ weizhengxian@sina.com
}

\author{
Guisheng Yin², Yingqi Wang ${ }^{2}$ \\ ${ }^{2}$ College of Computer Science and Technology Harbin \\ Engineering University, Harbin, China
}

\begin{abstract}
A human decision-making model is analyzed firstly. Based on this model, a decision-making efficiency model on control information system that is composed by information sustain, usable time, decision risk and level of degree is proposed in this paper. And then, in order to effectively design the control information system, based on analytic hierarchy process (AHP), an evaluation method of decision-making efficiency oriented multi-cooperated is established.
\end{abstract}

Keywords-control information system; decision efficiency; evaluation

\section{INTRODUCTION}

Control information system (e.g., train control information systems, electricity control information systems, ship electronic control information systems and so on) has the characteristics of both information systems and control systems. It is designed to provide efficient, proper, available information, so that users can make timely and accurate decisions and perform corresponding operations. In the context of the rapid development in information technology, overall synthesis and integration are the directions of the control information system, and when the human-computer interaction design for control information system is introduce, a problem which should be solved is to achieve the optimal matching between decision operating elements and tasks on the basis of information system design in order to conveniently use systems, reduce staff and promote efficiency. The aim of the control information system design is that people (users) and control information system can make full use of their respective advantages so that tasks can be quickly completed.

Decision-making efficiency is the efficiency of mutually coordinately and adaptively complete a specific task by characteristic factors of people and system in specific settings, where all factors such as people, system, environment and so on are employed, coordinated with each other according to some rules. Most control information systems are complex human-machine interaction systems which composed of multiple devices and coordinately used by many people. By evaluating decision-making efficiency of the control information system in an objective, accurate way, we can find

This work is sponsored by the National Natural Science Foundation of China under Grant No. 61502037, and the Fundamental Research Funds for the Central Universities under Grant No. 2014JJ009. the key factors of control information system, guide the design of control information system efficiently, optimize the configuration of information display and control resources, processing resources and people reasonably, use the people's labor load evenly, improve the availability of system information, and improve the holistic efficiency of control information system. In view of this, human behavior decision model is analyzed firstly, then human decision behaviors are divided into 6 types in terms of the different levels of informational support and stress. On the basis of it, a decisionmaking efficiency model, which consist of the level of informational support, time pressure, the risk of decisionmaking and the level of fatigue, is proposed according to domain characteristics in multi-people cooperated control information system. Finally, a decision-making efficiency evaluation model for the control information system based on the analytic hierarchy process is put forward in order to provide technical guidance and support for the design of the control information system.

\section{DECISION-MAKING EFFICIENCY MODEL FOR THE CONTROL INFORMATION SYSTEM}

\section{A. Decision behavior model for human}

In the control information system, information acquisition aims to help with decision making, the quality of decision making has a direct impact on the success of a task. Generally speaking, decision making is a process during which users make a reasonable judgment and formulate a right problem solution based on perceptive information intelligence (as shown in Figure 1).

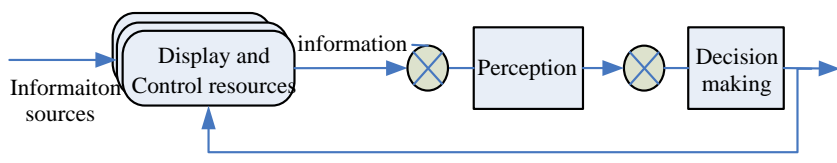

Fig. 1. Decision-making process diagram

Decision making is a behavior caused by human physiological reactions. It is extremely complicated for human physiological reactions and its impact on the effectiveness of the decision making behavior. Effectiveness of human 
decision making is varied with different external pressure. Initially, effectiveness of human decision making is very low, then it will be improved over time. When some threshold is surpassed, effectiveness of human decision making will be further decreased. It indicates that the effectiveness of human decision making under moderate pressure is the highest, while that under no pressure or excessive pressure will be decreased $^{[6,7]}$. The relation between effectiveness of human decision making and pressure is shown in Figure 2.

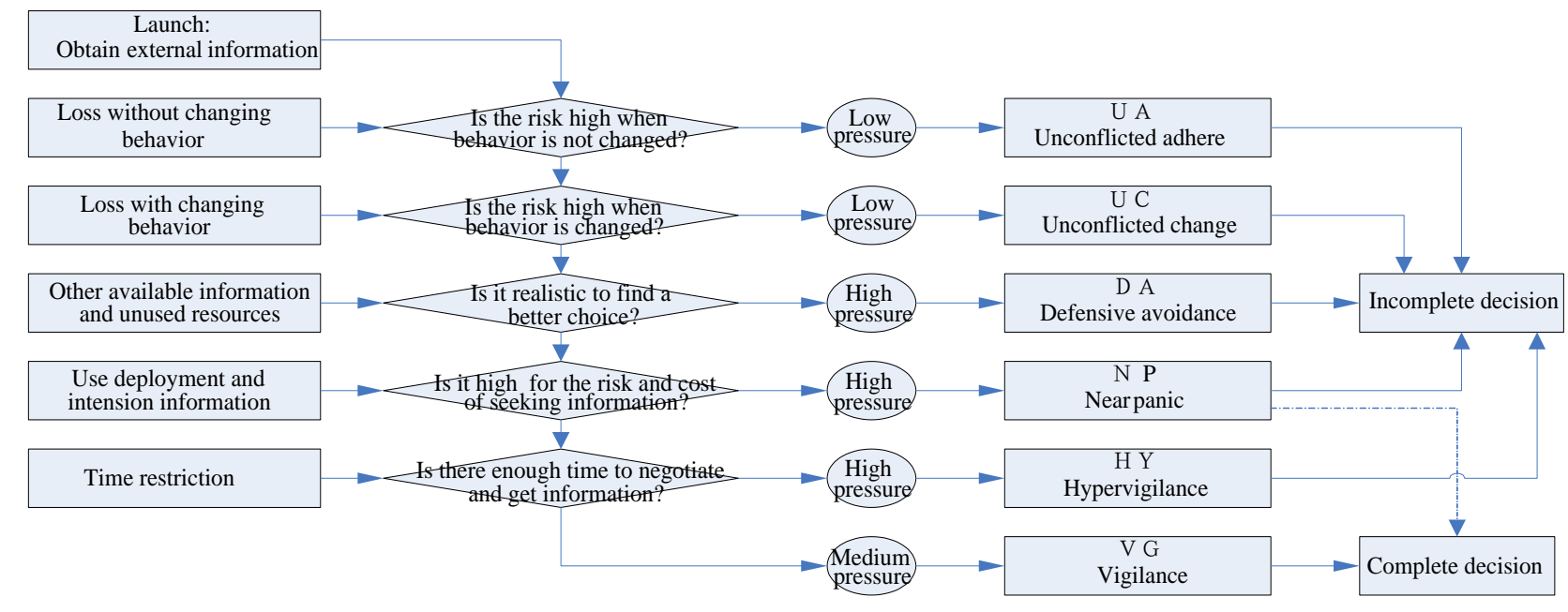

Fig. 2. Process of human operation and decision-making

The decision risk, time restriction and information do not entirely decide the pressure to be faced during the human decision making. And people's tension varies from pressure to pressure. Therefore we utilize the tension to measure the pressure to be faced during the human decision making. According to the tension, human decision making behavior may be grouped into following 6 types:

(1) Unconflicted adhere (UA): This kind of behavior mode makes decision-makers ignore the risk and make the decisions on their own.

(2) Unconflicted change (UC): This kind of behavior mode makes decision-makers change course without reservation.

(3) Defensive avoidance (DA): Decision-makers pass the buck in order to avoid the conflict.

(4) Near panic (NP): Decision-makers can't fully get complete information (i.e., enemy deployment and intention) so as to make a decision.

(5) Hypervigilance (HY): In order to get out of trouble, decision-makers take reckless action. This kind of status is also called 'panic'.

(6) Vigilance (VG): Decision-makers attempt to collect all relational information, so that they can make a decision after weighing the pros and cons.

More often than not, people keep the continuum of behaviors. If the risk of maintaining current situation is considered to be little (that is, maintaining current situation does not result in big loss), we call this behavior mode ' $U A$ ', and the tension is at a low in the UA. If the risk of changing behavior is considered to be little, this behavior mode is called 'UC', and the tension is at a low in the UC. If a better choice can be obtained from unused information, this behavior mode is called 'DA', and the tension is at a high in the DA. If complete information (for instance, system status or system development prediction) can't be obtained from unused information, this behavior mode is called 'NP', and the tension is at a high in the NP. If there is not enough time to negotiate and get information, this behavior mode is called ' $\mathrm{HY}$ ', and the tension is at a high in the HY. Otherwise, this behavior mode is called ' $\mathrm{VG}$ ', and the tension is at a high in the VG.

According above analysis, all types of behaviors but ' $\mathrm{VG}$ ' behavior are defective. The first two modes (that is, UA and UC) often come up together during the daily activities and indifferent decisions, while they will bring about wrong decision making during the important decisions. Likewise, DA and HY modes will make decision-makers easily suffer loss which could have been avoided. However, VG mode is appear in case of emergency easily and an optimal decision can be made in the VG. At the same time, It was considered that experts or trained people also can make an optimal or suboptimal decision, even though in the NP mode ${ }^{[8]}$.

In terms of human decision-making activities, the factors affecting decision-making efficiency can be divided into two aspects: subjective aspects and objective aspects. Subjective aspect are intrinsic factors of decision-making individual, such as personality traits, educational background, psychological quality, experience and so on, while objective aspects are extrinsic factors affecting decision-making, such as information, time, pressure and so on.

\section{B. Decision behavior model for the control information system}

Making a decision according to the relational information provided by control information system requires users to make an identification, judgment and decision within a period of time in terms of all information provided by system, then the decision-making results are fed back to system activities or 
processes, so that the expected results will be generated. Because carrying out tasks by most control information systems is a long, continuous process, which leads to users' psychological or physical tiredness. Moreover, control information systems require users make a decision in a very short time, which decision-making process and results may bring about very high risk. As a consequence, in terms of control information system design, decision-making efficiency for control information system must take into account four factors including the level of information support, time pressure, the risk of decision-making and the degree of fatigued based on the Figure 2 and the features of decisionmaking behaviors during the use of control information system. Figure 3 shows the relation between the decisionmaking efficiency of control information system and the influence factors (assumed that the individual quality, the use of literacy and training level in the control information system).

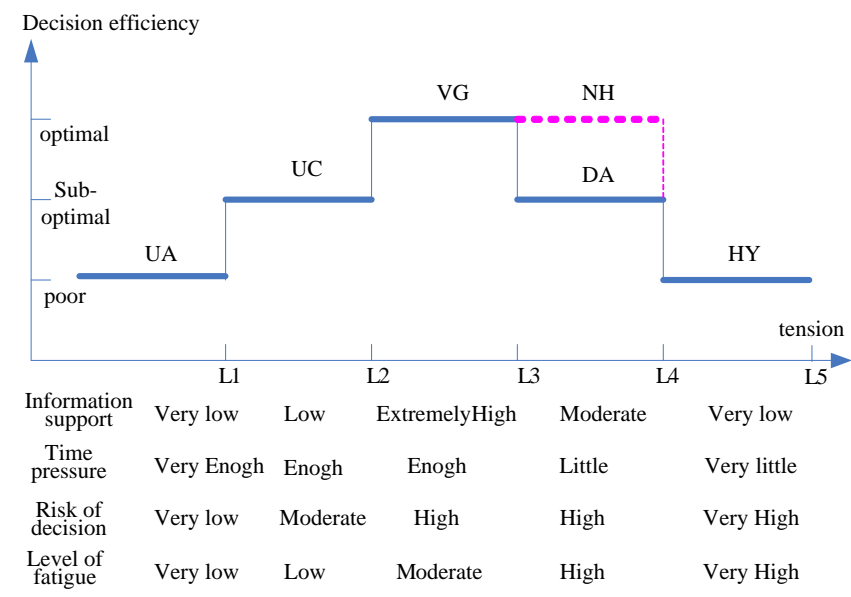

Fig. 3. Relation between influence factors and decision-making efficiency

When system performs a specific task, the users of system usually face relatively high level of decision-making risk. From Figure 3, we can see that our model qualitatively describes decision-making efficiency of control information system and relational influence factors. In order to guide the design of control information system efficiently, the effect of all influence factors on decision-making efficiency should be made quantitative assessment.

\section{DECISION-MAKING EFFICIENCY EVALUATION FOR CONTROL INFORMATION SYSTEM}

During the process of evaluate the decision-making efficiency for control information system, the influence factors are of great uncertainty, therefore analytic hierarchy process, or AHP for short, which is a simple, flexible and practical multi-criteria decision method proposed by T.L.Saat, can be utilized to evaluate the decision-making efficiency for control information system. AHP is be fit for some more complicated or fuzzy problems, especially for the problems which are hard to make a thorough quantitative analysis. A lot of researchers have joined AHP community since AHP was proposed by T.L.Saat. With more and more researchers focused on the AHP approach, it makes a rapid advancement. The main reasons are reasonable in theory and simple in application. The main features are to reasonable combine qualitative decision with quantitative decision and to make decision process be hierarchical and quantitative according to the thinking and psychological patterns.

A following calculation formula can be obtained based on AHP and Figure 3:

$$
E_{d}=w_{i} v_{i}+w_{t} v_{t}+w_{r} v_{r}+w_{f} v_{f}
$$

Where $w_{i}, w_{t}, w_{r}$ and $w_{f}$ represents the weight of the level of information support, time pressure, the risk of decisionmaking and the level of fatigue, respectively. The weights can be determined by expert evaluation method according to usage scenarios and task characteristics. $v_{i}, v_{t}, v_{r}$ and $v_{f}$ represents the parameter value of the level of information support, time pressure, the risk of decision-making and the level of fatigue, respectively.

The level of information support lies on individual information utilization degree and main relates with the ability to information processing (e.g., cognitive skills and efficiency), the ability to information transfer, the ability to individual information receiving and so on (as shown in Figure 4). In the setting of control information system, the level of information support are mainly the ability to information transfer and the ability to information receiving from the control information system design perspective, assumed that the cognition level difference among the people is ignored.

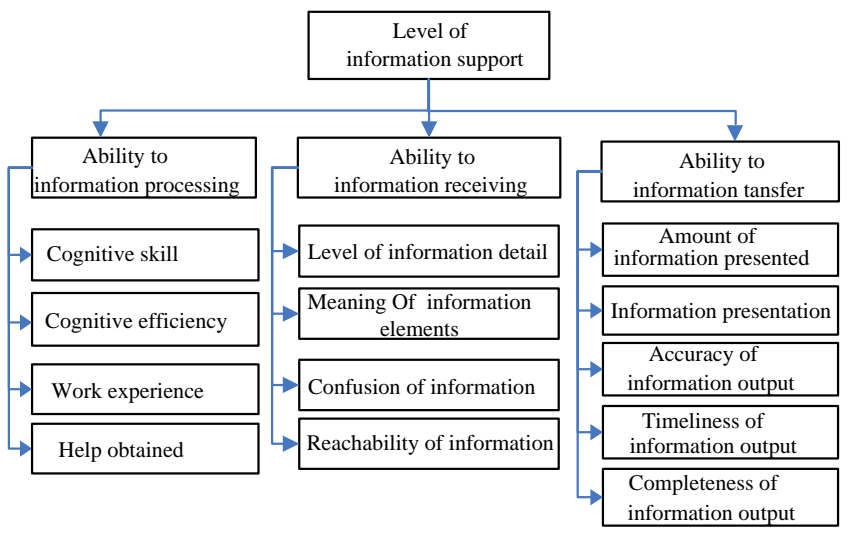

Fig. 4. Layered diagram of the level of information support

The ability to information receiving is mainly relevant with information quality, such as the accuracy (small error), the clarity (clear meaning and non-confusion) and the reachability of the information. For control information system, users can obtain information by virtue of the display and control equipments though the visual or auditory. For the audio auditory information from the display and control equipments of control information system, a display and control device can only receive one type of audio auditory information during a certain period, which the changes are small in different display and control device configurations of control information system. Therefore we do not consider it here. In the display and control device configuration, graphical display and data display of information usually present to users together, so that users make a corresponding decision or 
perform the corresponding operation activities. Because the differences range from the display and control device configurations of control information system and the use count of devices in different tasks, the amount of information presented to users once by the display and control devices is different during the process of carrying out task. If the relevant information can't be obtained once by the display and control devices, the other information can be gained by switching interfaces. So the following formula can be employed to evaluate the ability to information transfer of the display and control devices.

$$
E_{I}=w_{d} \frac{I_{d}}{I_{a}}+w_{o} \frac{I_{o}}{I_{a}}+w_{t} \frac{I_{t}}{I_{a}}
$$

Where $E_{I}$ represents information transfer efficacy (that is, the level of information support). $I_{a}, I_{d}, I_{o}$, and $I_{t}$ represents the number of information needed to get, the number of information obtained directly, the number of information obtained by one interface switching, and the number of information obtained by twice or more interface switching, respectively. $w_{d}, w_{o}$ and $w_{t}$ represents the corresponding weight, respectively.

Time pressure main depends on available time of decisionmaking, its formula is denoted as following:

$$
T p=T i /(T i+T a)=1 /(1+T a / T i)
$$

Where Ti represents the normal time of completing some specific decision task. Ta represents available time. In the above formula, if the value of $\mathrm{Ti}$ and the value of $\mathrm{Ta}$ are nearly equal to each other, the value of $\mathrm{Tp}$ is approximately equal to 0.5, which is fell into moderate level. As Ta increases gradually, there are enough time to complete a task, then the time pressure of completing a task decreases gradually. Whereas as Tp decreases gradually, Tp is close to 1 , then time pressure is relatively high.

In the control information system, the risk of decisionmaking varies from different tasks. For example, in the train control information system, the level of decision-making risk is moderate when the train is running normally. While the level of decision-making risk is very high when the train is running in the bad weather. Therefore the risk of decisionmaking is divided into five grades: no, low, moderate, high, very high. For different tasks, the corresponding level of decision risk can be gain by investigating users with rich experience.

The reasons for fatigue are various, including people's physiological cycle changes, tolerance for external stimulus, sleep, temperature, noise, labour intensity and so on. For the decision-making of control information system, labour intensity is an important factor. The higher the labour intensity is, the easier it will be to make people feel tired. When doubling the labor intensity, the level of fatigue will increase to 2.5-3 times, which indicates that labour intensity has a great impact on the emerge of fatigue. In the control information system, users' labor intensity varies from one configuration of the display and control devices to another, hence commanders' fatigues are different during the whole process of completing a task. The level of the fatigue is mainly obtained by investigating users with rich experience.

\section{CONCLUSIONS}

The complicated external environment is needed to deal with during the use of control information system. The pressure faced by the external environment needs to turn inward and to be bear by the corresponding users from different display and control devices of the control information system. Therefore personnel allocations generated by different display and control plans are different, which lead to the differences of decision risk, time pressure, the level of information support and so on faced by users, that of the number of tasks completed by users, and that of the level of fatigue. Based on the analysis of human behavior decision model, this paper proposes to evaluate the decision-making efficiency of control information system from four aspects of the level of information support, time pressure, decision risk and fatigue strength. Then the design of control information system and its display and control devices is guided from a decision-making efficiency point of view, aiming at optimizing the match between elements of information system and tasks, bringing into play their respective advantages of users and information system and complete the task quickly.

\section{REFERENCES}

[1] K.T.Yeo.Critical failure factors in information system projects. International Journal of Project Management, 2002,(20): 241-246.

[2] PoPo Poon,Christian Wagner.Critical success factors revisited: success and failure cases of information systems for senior executives. Decision Support Systems,2001,(30):393-418.

[3] Eldon Y.Li.Perceived importance of information system success factors A meta analysis of group differences. Information\&Management, 1997,(32):15-28.

[4] CHEN Feng, YI Shuping, YANG Wencai etal. Influence Mechanism of Matching on Human-information System Interactive Efficiency Within Entenprise Computerization Environment. Journal of Chongqing University, 2007, 30(10):36-41.

[5] NIU Zuocheng, WU Dewei, LEI Lei. Study on effectiveness evaluation for military equipment. ELECTRONICS OPTICS\&CONTRO, 2006,13(5):98-101.

[6] Irving L J, Mann L. Decision Making a Psychological Analysis of Conflict Choice and Commmitment, New York: the Free Press. 1998.57-89.

[7] Teigen K H. Yerkes-Dodson: A Low For All Seasons. Theory\& Psychology, 1994, 4(4): 525-547

[8] Klein G A, Orrasaru J, Calderwood R, Zsambok C E. Decision making inaction: Models and Methods. Norwood NJ:Ablex. 1993. 36-52.

[9] Hursh S R, Mcnally R E. Modeling Human Performance to Predict effectiveness. The new Frontier: Military and Civilian Application. 1993. 309-328.

[10] Kang Weiyong, Yuan Xiugan, Liu Zhongqi. Optimization design of vision display interface in plane cockp it based on mentalwork load. Journal of Beijing University of Aeronautics and Astronautics, 2008,34(7):782-785. 